\title{
Les syntagmes nominaux complexes dans les textes juridiques français
}

\begin{abstract}
With its point of departure in the verb valency theory, the present article introduces a classification of complex NPs in French legal texts. The heads of the NPs are subdivided into predicative and non-predicative nouns: predicative when they show the same valency pattern as verbs and non-predicative when they do not. The two classes may have bound and free expansions in the form of both complements and modifiers. The final classification of the NPs thus consists of eight different categories. The syntactic distinction between predicative and non-predicative nouns is supplemented, on the semantic level, by a gradable scale, ranging from the concrete to the abstract, along which the nouns are placed according to their degree of predication, i.e. according to the number of valents.
\end{abstract}

Si les grammairiens s'accordent pour définir l'aspect formel d'un syntagme nominal (déterminant - noyau - complément), les approches sont légion lorsqu'il s'agit de qualifier la nature des compléments possibles ou d'expliquer la relation qui unit ces compléments au substantif noyau.

Nous allons tenter de pallier cette carence de détermination univoque en transposant au domaine des syntagmes nominaux $(\mathrm{SN})$ les règles élaborées en matière de valence verbale et en postulant que les substantifs, comme les verbes, sont caractérisés par des expansions qui sont impliquées ou non par leur contenu sémantique.

Notre analyse s'inspire de la notion de valence telle qu'elle a été définie par Lucien Tesnière (1959) et de la théorie développée sur cette base par Michael Herslund et Finn Sørensen (1985, 1987, 1989 et 1992).

Tesnière conçoit le verbe comme le noeud de la phrase, le centre régissant autour duquel gravitent les autres unités, qu'il qualifie d'actants et 
de circonstants. Les actants désignent les êtres ou les choses qui, à un titre quelconque et de quelque façon que ce soit, même au titre de simples figurants, participent au procès exprimé par le verbe. Dans la phrase active, le prime actant correspond au sujet, le second actant à l'objet, et le tiers actant à celui au bénéfice ou au détriment duquel se fait l'action. Tout verbe a un nombre déterminé d'actants, lesquels sont toujours des substantifs ou des équivalents de substantifs. Les circonstants quant à eux, expriment les conditions de temps, de lieu, de manière, etc... dans lesquelles se déroule le procès. Leur nombre est indéfini et leur rapport au verbe est plus périphérique que celui des actants. Ce sont toujours des adverbes ou des équivalents d'adverbes.

La théorie de Tesnière, où actants et circonstants sont les subordonnés immédiats du verbe, comporte, selon Herslund et Sørensen, une faiblesse majeure, à savoir l'absence de critère opérationnel permettant d'effectuer une distinction mécanique et univoque entre les deux catégories d'unités lorsque celles-ci présentent une identité formelle, comme dans le cas des syntagmes prépositionnels. Dans le cadre d'une étude visant à une classification des verbes français, Herslund et Sørensen proposent par conséquent d'établir une nouvelle distinction fondamentale entre d'une part les membres de phrase qui sont déterminés par le verbe, et d'autre part ceux qui sont ajoutés à la phrase entière. Parmi les premiers, ils distinguent entre les actants à proprement parler et différentes sortes de modifieurs adverbiaux du type "sa conférence a duré 3 heures". Seuls les actants définissent la valence du verbe. Ils se subdivisent en trois classes:

- l'actant fondamental qui constitue avec le verbe un prédicat syntaxique (à savoir l'objet d'un verbe transitif ou le sujet d'un verbe intransitif),

- un deuxième actant qui se combine avec ce prédicat pour former une prédication (il s'agit soit du sujet d'un verbe transitif, soit du sujet d'un verbe intransitif - ce dernier constitue donc, avec le verbe, aussi bien le prédicat que la prédication),

- un troisième actant qui exprime à la fois une relation au verbe et à un autre actant (qui peut être l'objet, le sujet d'un verbe intransitif, mais jamais le sujet d'un verbe transitif). Cet actant, qualifié d'objet indirect ou d' "adjet", recouvre dans la grammaire traditionnelle aussi bien les fonctions du 
complément d'objet indirect (ou objet prépositionnel) que celles du complément d'attribution, du complément local, et celles des attributs du sujet et de l'objet.

Parmi les membres de phrase qui sont ajoutés à la phrase entière, ou plutôt à la prédication, Herslund et Sørensen comptent en premier lieu les circonstants à proprement parler, à savoir les indications de temps et de lieu, dont la quantité n'est pas spécifiée par le contenu lexical du verbe. En second lieu, et bien qu'ils n'y consacrent pas une analyse approfondie, les auteurs discernent, parallèlement à la distinction opérée au sein des unités déterminées par le verbe, différents types de modifieurs adverbiaux libres qui, sans entrer dans la relation prédicative, qualifie celle-ci en précisant sa modalité ou en exprimant une qualité qui la caractérise (ex. il mange vite).

Nous fondant sur les définitions données ci-dessus, nous poserons que les substantifs français, suivant qu'ils établissent ou non un rapport verbal avec une ou plusieurs de leurs expansions, se répartissent en deux classes: les substantifs prédicatifs, qui reproduisent les schémas vvalentiels des verbes ou des locutions verbales dont ils sont dérivés ou avec lesquels ils ont une certaine parenté, et les substantifs non prédicatifs, qui sont tous les autres.

Nous énoncerons comme hypothèse de départ que, de la même manière que les verbes peuvent se rencontrer avec des membres de phrase déterminés par leur contenu lexical et d'autres qui sont ajoutés à la prédication, les substantifs peuvent s'employer avec des expansions qui sont impliquées par leur contenu sémantique, que nous appellerons essentielles, et des expansions qui ne dépendent pas de ce contenu, que nous qualifierons de circonstancielles. Ces dernières peuvent s'associer aux deux classes de substantifs et se combiner indéfiniment avec d'autres expansions.

Les expansions sont, à leur tour, de deux sortes: les compléments, c'està-dire les entités essentielles et circonstancielles ayant un certain 
rapport avec le substantif, et les modifieurs, à savoir les unités qui, d'une manière ou d'une autre, caractérisent le substantif ${ }^{1}$.

Cette triple distinction nous permet d'établir le schéma suivant:

\section{$\underline{\text { Tableau } 1}$}

Il ressort de ce tableau que les expansions possibles dans un SN complexe sont au nombre de huit. Toutefois, il convient de souligner dès à présent que le rôle de ces expansions quant à la distinction fondamentale entre les deux classes de substantifs varie considérablement. Ainsi que le révélera l'étude que nous allons entreprendre ci-dessous, seuls les compléments essentiels de la catégorie I.A.1. confèrent aux substantifs leur caractère prédicatif.

1 Si nous avons emprunté le terme de modifieur à Herslund et Sørensen, les expressions de compléments essentiels et de compléments circonstanciels, dont nous ferons usage dans ce qui suit, ont été utilisées à l'origine par Wagner et Pinchon (1962). Elles correspondent chez eux à la distinction entre les actants et les circonstants de Tesnière et de la grammaire valentielle. Nous les emploierons quant à nous dans un sens plus vaste puisque ces compléments, comme nous le verrons, peuvent s'associer aussi bien avec des substantifs prédicatifs qu'avec des substantifs non prédicatifs. 
Nous avons travaillé à partir d'un corpus de textes juridiques, et plus spécialement de contrats, où par tradition le besoin de précision et de concision a rendu l'emploi des SN complexes particulièrement fréquent. En effet, les substantivations évitent souvent d'avoir recours à des propositions relatives et, plus généralement, à des phrases construites à base de verbes finis $^{2}$. Nous avons analysé 400 syntagmes au total, soit l'ensemble des SN complexes de quatre contrats d'intermédiaires commerciaux. Précisons que parmi les SN complexes, nous nous intéresserons principalement aux noyaux comportant comme expansions soit des syntagmes prépositionnels, soit des syntagmes participiaux, soit encore des propositions relatives. Ces expansions, en raison de leur fréquence et de leur structure, se prêtent remarquablement à une investigation visant à définir le style nominal dans le discours juridique français ${ }^{3}$. Nous ne mentionnerons que pour mémoire, ou parce qu'ils accompagnent les expansions précédentes, les adjectifs, locutions adjectivales et substantifs épithètes et les cas plus rares de propositions concessives, appositionnelles et infinitives.

2 Sur l'importance et l'abondance des substantivations dans le langage du droit, voir Sourioux et Lerat (1975) et Cornu (1990). Notons que nous considérons comme non pertinente pour la présente analyse la délimitation des contrats par rapport aux autres catégories de textes juridiques.

3 Elles se prêteront de même à une analyse contrastive tendant à mettre à jour les spécificités de ce style nominal dans deux communautés linguistiques distinctes (voir article à paraître). 
Notre étude s'articulera à partir de la nécessité d'élaborer une distinction opérationnelle entre substantifs prédicatifs et substantifs non prédicatifs: dans une première partie, nous déterminerons quelles sont les expansions qui confèrent

$\mathrm{s}$ leur caractère prédicatif, et dans une seconde partie, nous nous attacherons à délimiter les expansions non valentielles des $\mathrm{SN}$ complexes.

\section{Détermination des expansions valentielles}

Les expansions valentielles sont les compléments essentiels des substantifs prédicatifs (voir tableau 1, I.A.1). Elles correspondent aux actants des verbes et, tout comme les actants définissaient la valence verbale au sein de la phrase, les expansions valentielles vont définir la va-lence du substantif au sein du syntagme nominal. Selon leur rôle, elles seront qualifiées de sujet, $\begin{array}{llllll}\text { d'objet ou d'objet indirect }(\mathrm{S}, \quad \mathrm{O}, & \mathrm{OI}) \text {. Deux }\end{array}$ 
facteurs vont déterminer l'existence et la nature de ces expansions: les divers types de nominalisations et les différentes sortes de dérivations.

\subsection{Les types de nominalisations}

Le substantif prédicatif peut exprimer trois sortes de nominalisations: nucléaire, subjective ou objective 4 . Dans le premier cas, qui est le plus fréquent, la nominalisation désigne le procès exprimé par le verbe (ex. accepter - acceptation; résilier - résiliation, etc...). Dans le second cas, la nominalisation désigne le sujet, c'est-à-dire celle/celui/ce qui réalise l'action exprimée par le verbe (ex. vendre - vendeur; diriger - directeur ou dirigeant). Dans le troisième cas enfin, la nominalisation correspond à l'objet: direct (ex. accuser - accusé; facturer - facture), ou indirect (ex. destiner - destinataire).

Alors que certains verbes ont deux nominalisations différentes selon qu'il s'agit d'une action ou de son résultat:

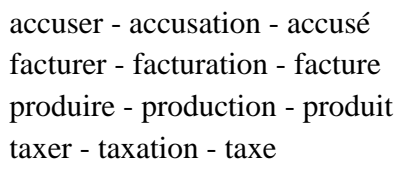

il en va différemment des cas où les nominalisations nucléaire et objective sont identiques d'un point de vue formel:

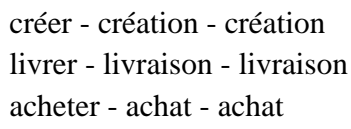

Les nominalisations du type création, livraison ou achat - c'est-àdire dérivées de certains verbes perfectifs et transitifs (bivalents ou trivalents) - sont alors ambiguës, dans l'absolu, puisqu'elles peuvent désigner aussi bien l'action verbale proprement dite que l'objet de cette action (créer/livrer/acheter quelque chose) et donc le résultat concret d'un processus ${ }^{5}$. Cette ambiguiité sera levée par le contexte linguistique. En effet, il est important de souligner qu'une nominalisation nucléaire peut impliquer un ou plusieurs actants, selon la valence du verbe auquel elle correspond, alors qu' une nominalisation objective qui englobe, outre l'action verbale, un des actants - à savoir l'objet - ne pourra s'associer qu'un actant autre que celui qu'elle désigne, soit le sujet ou éventuellement un objet indirect, mais en aucun cas un objet (cf. Ulland 1991, 8). Par conséquent, la présence d'un complément d'objet à côté d'une nominalisation exclut la possibilité qu'il puisse s'agir d'une nominalisation objective. Il ne pourra alors être question que d'une nominalisation nucléaire désignant une action verbale et formant un prédicat avec son objet 6 . Par exemple, si livraison est ambigu, la

\footnotetext{
4 Voir à ce propos Ulland (1991, 4-11) et Stage (1986, 211).

5 Nous nous contentons ici de constater l'existence de cette polysémie. La question, d'ordre lexical, de savoir quels sont les verbes qui acceptent des nominalisations nucléaire et objective différentes et quels sont ceux qui admettent des nominalisations identiques, ou qui ne permettent pas toutes les nominalisations possibles, suppose des investigations qui excèderaient le cadre de cette étude.

6 Cette observation ne s'applique qu'aux substantifs directement dérivés de verbes pleins, et non aux cas où étymologiquement, le nom a précédé le verbe, ou à ceux où les substantifs correspondent aux noms contenus dans des constructions à verbe support (voir infra).
} 
livraison des marchandises ne peut traduire qu'une action. De même, signature est ambigu, mais non la signature du présent contrat 7 .

Si l'adjonction d'un complément d'objet permet ainsi de désambiguïser certains cas de nominalisations polysémiques, il nous faut mentionner toutefois qu'une équivoque subsiste, que seule une analyse approfondie du contexte extralinguistique permettra de lever. Il s'agit des situations où le contenu sémantique d'une nominalisation nucléaire implique un complément objectif, et où la forme de cette construction est identique à la nominalisation objective suivie d'un complément subjectif:

l'achat de la société Y

Il peut s'agir ici soit de $\mathrm{X}$ qui achète la société $\mathrm{Y}$ (donc d'un procès), soit de l'objet acheté par la société Y (donc le résultat concret). L'ambi-

7 D'autres procédés de désambiguïsation existent également, que nous n'approfondirons pas dans la mesure où nous nous consacrons ici à une analyse interne des SN, c'est-à-dire aux rapports existant entre le substantif noyau et ses expansions. Mentionnons toutefois, pour mémoire, qu'on se trouvera en présence d'un résultat concret toutes les fois où une nominalisation figurera comme sujet d'un verbe d'état ou comme complément d'un verbe impliquant un objet concret (ex. la signature figure/se trouve au bas de la page; il a trouvé sa livraison sur le port). De plus, l'emploi du pluriel indique souvent - mais pas toujours - qu'il s'agit de choses concrètes (ex. les achats de la société). Inversement, sera nucléaire une nominalisation employée avec un adjectif énonçant une modalité du nom - et qui correspond à un adverbe qualifiant un verbe (ex. une livraison rapide/livrer rapidement). 
guïté consiste en l'impossibilité de distinguer, au niveau de la structure linguistique, entre un procès et le résultat concret d'un procès, et non entre deux procès. En effet, le sujet d'une nominalisation nucléaire serait introduit au moyen de la préposition par, et peut d'ailleurs, le cas échéant, être associé avec l'objet. Les substantifs déverbaux tels que achat, c'est-à-dire dérivés de verbes perfectifs, transitifs et trivalents, se combinent par conséquent avec les compléments suivants:

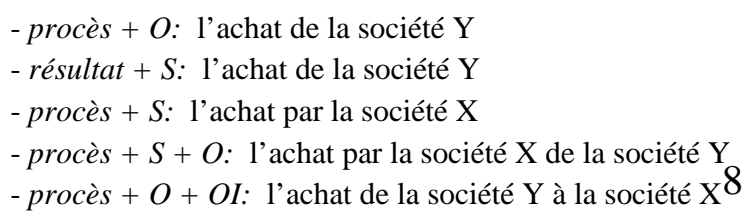

Soulignons pour terminer, et bien qu'il ne s'agisse pas ici de lever une ambiguïté, qu'une nominalisation subjective, qui contient à la fois le verbe et son sujet, ne peut être suivie que d'un complément objectif (ex. les dirigeants de la société). De même, une nominalisation correspondant à l'objet indirect, et qui comporte à la fois le verbe et un de ses actants, soit l'objet indirect, ne peut être associée qu'à un complément subjectif ou objectif (ex. le mandataire de M. Vasseur; le destinataire des marchandises).

Ces différentes formes de nominalisations ne s'observent que parmi les substantifs directement dérivés de verbes, ainsi que le montrera la description des divers types de dérivations que nous allons aborder à présent.

\subsection{Les types de dérivations}

Le substantif noyau sera soit dérivé d'un verbe plein, soit dérivé d'une locution verbale, soit encore il appartiendra à la même aire sémantique qu'un verbe dont il n'est pas dérivé directement.

8 Bien que théoriquement possibles, les cas de syntagmes nominaux comportant trois compléments valentiels (ex. l'achat par la société X de la société $Y$ à la société Z) sont extrêmement rares (voir également Stage 1986, 210). 


\subsubsection{Le substantif noyau du $\mathrm{SN}$ est dérivé d'un verbe}

Les compléments essentiels seront alors identiques à ceux du verbe initial, et comme celui-ci, les substantifs seront monovalents, bivalents ou trivalents.

- Le substantif noyau peut être caractérisé par un complément subjectif sous forme de syntagme prépositionnel, où le régime de la préposition correspondrait au sujet d'une phrase active correspondante:

la durée/la fin/l'expiration du contrat

l'évolution des tarifs de la société $X$

les désirs des clients

la confirmation préalable et écrite de la société

la première demande de la société

l'achat $d u$ concessionnaire (résultat)

l'achat par le concessionnaire (procès)

la collaboration entre la société $X$ et la société $Y$

La préposition employée est de, accessoirement entre si le verbe dénote une relation entre deux sujets, et par s'il y a homonymie entre les nominalisations objective et nucléaire (voir 1.1.).

- Le substantif noyau peut être caractérisé également par un complément objectif sous forme d'un syntagme prépositionnel où le régime de la préposition correspondrait à l'objet de la phrase active correspondante:

l'acceptation de l'ordre

la rédaction du contrat en langue française

l'exercice des fonctions telles que définies au présent contrat

la commercialisation des produits dont la vente lui est confiée

le placement des articles suivants

l'extension de son champ d'activité

la livraison des marchandises, objets de ces ordres

la direction effective de la société $Y$

les dirigeants de la société $X$

des achats indépendants de pièces de rechange et d'accessoires

La préposition employée est de, comme c'était le cas pour les compléments subjectifs. Il est à noter toutefois que si les deux types de compléments figurent comme expansions du même noyau, l'objet demeurera exprimé par de suivi du régime, alors que le sujet sera introduit au moyen de la préposition par (voir aussi 1.1.):

l'encaissement total de la facture par le concédant

le refus par l'agent d'accepter ces modifications

Le complément objectif peut à son tour comporter une expansion sous forme de syntagme prépositionnel, complément d'objet, qui est en quelque sorte situé à un second niveau de profondeur :

une cause de rupture $d u$ présent contrat

la concession exclusive de la vente de ses machines-outils

Il peut parfois être difficile de déterminer s'il s'agit du complément objectif ou subjectif d'une nominalisation nucléaire. Lorsque le référent du substantif régime de la préposition pourrait être aussi bien l'objet que le sujet de l'action verbale, seule une analyse du contexte extralinguistique permettra de se prononcer définitivement à ce sujet: 
l'agrément du successeur 9

Mentionnons également que les compléments essentiels des substantifs dérivés n'ont pas besoin de figurer expressément dans le syntagme. Ils peuvent très bien n'être présents qu'à l'état latent. Ainsi dans l'exemple les ventes directes sur le secteur, les compléments subjectif, objectif et d'objet indirect sont tous sous-entendus.

- Le substantif noyau peut être caractérisé finalement par un complément d'objet indirect sous forme d'un syntagme prépositionnel où le régime de la préposition correspondrait à l'objet indirect de la phrase active correspondante:

son consentement à toute succession qui lui est présentée (objet prépositionnel)

leur vente à la clientèle (complément d'attribution)

une meilleure pénétration sur le marché danois (complément local)

Cet objet indirect peut être combiné, à l'intérieur du syntagme nominal, avec un autre complément:

l'extension de son champ d'activité sur de nouveaux articles ou de nouvelles marques

9 Cette difficulté concerne les cas où il n'existe pas de nominalisation objective correspondante (l'entité dénotée est en elle-même trop abstraite). En effet, si une telle nominalisation existait, la construction $p a r+S$ de la nominalisation nucléaire exclurait l'équivoque (voir 1.1.). 


\subsubsection{Le substantif noyau du $\mathrm{SN}$ est dérivé d'une locution verbale}

Il s'agit de locutions verbales construites à l'aide de verbes supports plus ou moins vides de sens, telles que prendre l'initiative, mettre en cause, avoir droit, avoir l'intention, faire un relevé. Le plus souvent, le substantif noyau correspondra en fait au nom qui est contenu dans la construction à verbe support. Nous associerons à ces locutions verbales les prédications avec être suivi d'un adjectif, telles que être responsable, être défectueux.

Comme précédemment, les compléments essentiels seront semblables à ceux des locutions verbales correspondantes. Ils seront subjectifs:

l'initiative de la société concédante

la responsabilité $d u$ concédant

la défectuosité du matériel

\section{objectifs:}

la mise en cause de la responsabilité de la société mandante

le relevé mensuel des commissions

ou objets indirects:

le droit à la commission

son intention de ne pas renouveler le présent contrat

Ces objets indirects peuvent à leur tour constituer l'expansion d'un complément objectif et donc se situer à un deuxième niveau de profondeur:

la présentation d'un nouveau candidat à la succession

\subsubsection{Le substantif noyau a une certaine parenté avec un}

Certains substantifs, bien que non dérivés directement de verbes ou de locutions verbales, peuvent se voir associer des expansions valentielles et sont partant à considérer comme des substantifs prédicatifs.

Parfois le substantif appartient à la même aire sémantique qu'un verbe plein:

terme (finir)

accord (approuver)

autorité (commander, dominer)

texte (écrire)

avenant (modifier)

dommages et intérêts (indemniser)

Parfois le verbe a existé dans un état antérieur de la langue mais ne subsiste plus aujourd'hui, ou bien est en voie de disparition. Le substantif peut alors se rencontrer comme composante d'une locution verbale:

prix (priser - fixer le prix de)

Comme pour les substantifs déverbaux, les compléments peuvent être subjectifs:

le terme du contrat

l'accord de la société

l'autorité des dirigeants de la société X ou de leurs délégués

les prix courants $d u$ concédant

ou objectifs: 
le texte de la convention à intervenir entre l'agent ou ses ayants droit et le

successeur éventuel

ou objets indirects:

un avenant au présent contrat

des dommages et intérêts pour la société

et être combinés entre eux:

les prix de vente de la société concédante au concessionnaire

Nous venons de décrire les compléments valentiels des substantifs, et les facteurs qui déterminent leur emploi et leur caractère. Il nous faut à présent classer les autres expansions possibles dans les SN complexes.

\section{Délimitation des expansions non valentielles}

Bien que ces expansions n'influent pas sur le caractère prédicatif des substantifs auxquels elles sont associées, on peut néanmoins les répartir en deux classes, suivant que le lien qui les unit au terme régissant est essentiel ou circonstanciel.

\subsection{Les expansions essentielles}

La présence de ces expansions n'est pas fortuite, en ce qu'elle est déterminée par le contenu sémantique du substantif noyau. On a donc, parallèlement à la relation valentielle décrite précédemment, un rapport d'un autre ordre, tout aussi étroit, mais ne présentant aucunement un caractère de prédication. Une analyse des différentes expansions essentielles illustrera ce qu'il y a lieu d'entendre par là.

\subsubsection{Les compléments essentiels des substantifs non pré}

Nous rangerons dans ce groupe les syntagmes prépositionnels compléments établissant soit une relation, plus ou moins figurée, de la partie au tout, soit une relation de la catégorie à un de ses éléments. Dans le premier cas, nous parlerons de compléments "partitifs", dans le second, de compléments de "définition".

\subsubsection{Les compléments "partitifs"}

Le référent du substantif noyau représente un élément, une composante, une partie (qui peut se révéler être la partie totale, l'ensemble) du référent du régime de la préposition:

dix pour cent $d u$ montant des factures

la moitié au moins du capital de ladite société

l'ensemble du territoire concédé

au dernier jour de chaque mois

la personnalité de Monsieur C.D.

Nous intégrons dans cette série les cas un peu spéciaux où le terme régissant du SN est en fait le complément circonstanciel, ou essentiel, du 
terme subordonné - ce que révèle d'ailleurs une inversion de la structure. Le second substantif est en général un nom déverbal:

le jour de la signature du présent contrat (la signature du 3.2.1992)

la date de la signature du présent contrat (la signature du 3.2.1992)

la date de la commande (la commande du 3.2.1992)

\subsubsection{Les compléments de "définition"}

Lorsque le référent du substantif noyau représente une "catégorie" à laquelle appartient le référent du syntagme prépositionnel complément, on peut établir une relation d'inclusion et d'identité entre les deux substantifs, analogue à celle existant entre hyperonyme et hyponyme:

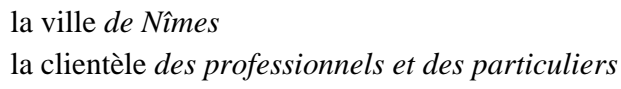

\subsubsection{Les modifieurs essentiels des substantifs prédicatifs et}

Les modifieurs essentiels forment un genre un peu particulier d'expansions, correspondant non pas aux actants traditionnels des verbes - S, $\mathrm{O}$, OI - et partant aux compléments essentiels de leurs formes nominales, mais constitué de constructions adverbiales impliquées par le contenu sémantique de certains substantifs:

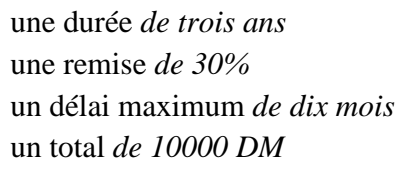

\subsection{Les expansions circonstancielles}

Nous reprendrons ici également la distinction esquissée en introduction entre compléments et modifieurs.

\subsubsection{Les compléments circonstanciels (tableau 1, I.B.3. et}

Il s'agit d'entités ayant un certain rapport avec le substantif, mais leur présence, comme celle des circonstants auprès des verbes, n'est pas impliquée par le contenu sémantique du noyau. Elles peuvent par conséquent, à l'exception des compléments de possession (voir infra), s'associer indifféremment aux substantifs prédicatifs et aux substantifs non prédicatifs, tout comme elles peuvent se combiner à l'infini avec d'autres expansions. Il s'agit de syntagmes prépositionnels décrivant les circonstances de temps, de lieu, de manière, etc... dans lesquelles se déroule le procès, ou qui constituent le cadre situationnel où se situe le référent du substantif noyau. La nature de la préposition varie avec la circonstance décrite.

- Voici d'abord quelques exemples de compléments circonstanciels de substantifs prédicatifs:

la durée d'un an à compter de ce jour

le prix de la facturation lors de l'achat

un préavis réciproque de 4 mois 
la vente sur le territoire français des produits visés ci-dessous à l'article 2

le décret $\mathrm{n}_{-}$58-1345 du 23 décembre 1958 relatif aux agents commerciaux

la résiliation du présent contrat aux torts de l'agent, moyennant dommages et

- Quand aux substantifs non prédicatifs, ils ont d'abord des compléments circonstanciels tout à fait analogues à ceux des substantifs prédicatifs:

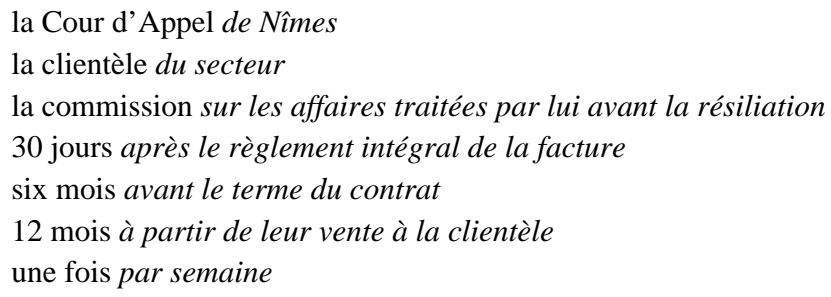

mais ils ont aussi une catégorie un peu particulière de compléments de "possession". Ces compléments recouvrent une relation d'appartenance qui, dans une phrase active, serait exprimée à l'aide du verbe avoir (au sens de posséder) avec un sujet présentant le trait [+hum]:

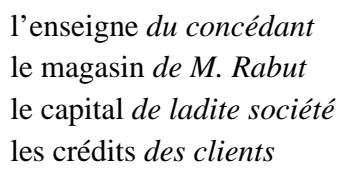

\subsubsection{Les modifieurs circonstanciels (tableau 1, I.B.4. et}

Il s'agit d'adjectifs, de locutions adjectivales et de substantifs épithètes, de syntagmes prépositionnels de type prép $+\mathrm{N}$, de syntagmes participiaux et de propositions relatives, concessives, appositionnelles et infinitives qui modifient le substantif noyau en y adjoignant une propriété ou une qualité. Ces modifieurs, tout comme les compléments circonstanciels décrits plus haut, ne sont pas spécifiés par le contenu sémantique du substantif noyau. Ils peuvent qualifier un substantif prédicatif tout comme un substantif non prédicatif. Quant à leur nombre, il n'est pas limité: dans un syntagme nominal, il peut n'y en avoir aucun, comme ils peuvent se combiner indéfiniment, entre eux ou avec d'autres expansions.

\subsubsection{Adjectifs, locutions adjectivales et substantifs épithètes}

Ils peuvent modifier aussi bien un substantif prédicatif:

les ventes directes sur le secteur

un prix supérieur à celui fixé par ces tarifs

qu'un substantif non prédicatif:

le chiffre d'affaires annuel

des entreprises concurrentes du concédant

les produits objets du présent contrat

\subsubsection{Syntagmes prépositionnels de type prép+N}

- La préposition sera typiquement $d e$, plus rarement avec, et la relation entre $\mathrm{N} 1$ et $\mathrm{N} 2$ est de telle sorte qu'on peut assimiler, quant au degré de 
cohésion et à l'unité sémantique, le syntagme [N1 prép N2] à un nom composé, que le substantif noyau soit prédicatif:

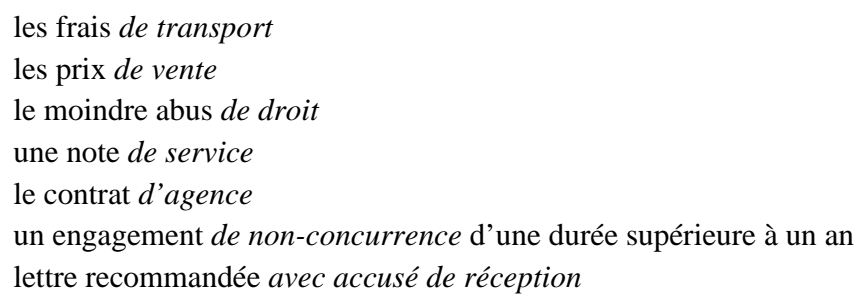

ou non prédicatif:

la Cour d'Appel

l'agent de commerce

les pièces de rechange

son champ d'activité

le bon de garantie

le chiffre d'affaires annuel

- Nous rapprocherons de ce genre de modifieurs les syntagmes prépositionnels classificatoires construits sur les modèles suivants: de + possessif $+\mathbf{N}$, où l'adjectif possessif reproduit un actant du nom prédicatif qu'il précède, de + tout/ce/cette $+\mathbf{N}$ et de + déterminant indéfini + locution adjectivale $+\mathbf{N}$ :

la personne de son choix

les accidents de toute nature

des machines-outils d'une autre marque que celle de la société Outima

- Les compléments essentiels associés à l'ensemble [N1 prép N2] peuvent être les $\mathrm{S}$, $\mathrm{O}$ ou OI du substantif noyau ou du substantif régime lorsque les deux sont prédicatifs:

la liquidation de biens $d u$ concédant

les conditions de collaboration entre la Société X et la Société $Y$

le service après vente des machines vendues par ses soins

aucun droit de gage sur ceux-ci

Lorsque seul le substantif régime est prédicatif, il peut lui aussi avoir des compléments essentiels (S-O-OI) qui s'associeront pareillement à l'ensemble [N1 prép N2]:

la date d'expiration de la période en cours

le point de départ $d u$ préavis

le délai normal de rotation de ce stock

la date de notification de la résiliation du présent contrat

\subsubsection{Syntagmes participiaux}

- On rencontre des participes passés et des participes présents aussi bien comme expansions de substantifs prédicatifs:

les conditions fixées au présent contrat

les affaires traitées par lui avant la résiliation

les révisions et réparations assurées par le bon de garantie délivré lors de la vente de chaque machine

une indemnisation intervenant dans les conditions prévues ci-dessus

des annulations provenant de retards imputables au concédant 
tout litige pouvant survenir dans l'interprétation ou l'exécution du présent

contrat

des achats indépendants de pièces de rechange et d'accessoires s'élevant à un

total de plus de $10000 \mathrm{DM}$

que comme expansions de substantifs non prédicatifs:

les machines vendues par ses soins

les produits représentés par l'agent dans le cadre des droits que lui donne le

présent contrat

un client situé dans le secteur concédé au concessionnaire

une banque française choisie par les parties

le bon de garantie délivré lors de la vente de chaque machine

les produits faisant l'objet du contrat

le mois suivant l'arrêté des comptes

- Parfois, l'exigence de précision des textes juridiques conduit à exprimer le sujet ou le destinataire logiques de l'action contenue dans un substantif déverbal perfectif non comme des compléments essentiels, mais comme des composantes de modifieurs circonstanciels sous forme de syntagmes participiaux:

toute livraison faite par la Société concédante à la SARL

toutes les commandes fermes passées par le concessionnaire

les remises ou ristournes accordées aux clients

Le sujet ou l'objet indirect étant contenus dans le syntagme participial, le substantif noyau perd sa faculté de se voir associer, selon le cas, un complément subjectif ou un objet indirect. Etant ainsi privé de certains de ses compléments valentiels, il acquiert du même coup un sens résultatif plus concret.

Il semble d'ailleurs que le fait d'associer un syntagme participial au substantif noyau tende à exclure la faculté de ce dernier de se voir adjoindre des compléments valentiels. Nous n'avons en effet trouvé, dans l'ensemble de notre corpus, qu'une seule occurrence, un peu particulière, qui contredise cette tendance: un accord de sa part valant approbation .

\subsubsection{Propositions relatives}

Il s'agit de différentes sortes de propositions relatives adjectives qui, ici aussi, qualifieront indifféremment les substantifs prédicatifs:

toute condamnation qui serait prononcée contre lui de ce chef

les commandes qui lui seront passées par M. Rabut 
les instructions que lui font parvenir les dirigeants de la société ou leurs délégués

les accidents de toute nature dont l'agent ou ses préposés seraient victimes

et les substantifs non prédicatifs:

l'année qui suivra son expiration

les pièces de rechange fournies par elle qui resteraient en stock dans le magasin de M. Rabut

le secteur qu'il a proposé comme étant celui dans lequel il travaille

habituellement

les produits dont la vente lui est confiée

les produits sur lesquels portait son activité

\subsubsection{Propositions concessives, appositionnelles et infinitives}

Elles qualifieront surtout les substantifs prédicatifs:

la fin du contrat, quelle qu'en soit la cause, et quel qu'en soit le moment

le montant net des factures, hors toutes taxes présentes et futures

la convention à intervenir entre l'agent ou ses ayants droit et le successeur

éventuel

En ce qui concerne les substantifs non prédicatifs, nous n'avons rencontré que des appositions:

le pays d'exécution, soit la France

une commission brute sur le chiffre d'affaires annuel après déduction de toutes

taxes, escomptes, remises, avoirs, frais de transport et d'emballage

\section{Conclusion}

L'analyse que nous venons de faire révèle le caractère disparate des expansions pouvant être contenues dans un SN complexe et la nature variée des liens unissant ces expansions au substantif noyau. Parmi ces liens, nous considérons comme primordiale la relation existant entre les substantifs prédicatifs et leurs compléments essentiels (tableau 1, I.A.1.), en ce que seuls ces derniers confèrent au terme régissant son caractère prédicatif. Nous pouvons à présent développer et compléter le tableau 1, présenté en introduction, de la manière suivante:

\begin{tabular}{|c|c|c|}
\hline & $\begin{array}{ll}\text { A } & \\
\cdot & \\
& \text { Expansions } \\
& \text { essentielles } \\
\end{array}$ & $\begin{array}{ll}\text { B } & \\
\cdot & \\
& \text { Expansions } \\
& \text { circonstancielles } \\
\end{array}$ \\
\hline & $\begin{array}{l}1 \\
\text { ) } \\
\underline{\mathrm{e}} \\
\underline{\mathrm{s}} \\
\underline{\mathrm{s}} \\
\underline{\mathrm{e}}\end{array}$ & $\begin{array}{ll} & \\
& \\
\text { Compléments } \\
\text { circonstanciels }\end{array}$ \\
\hline
\end{tabular}




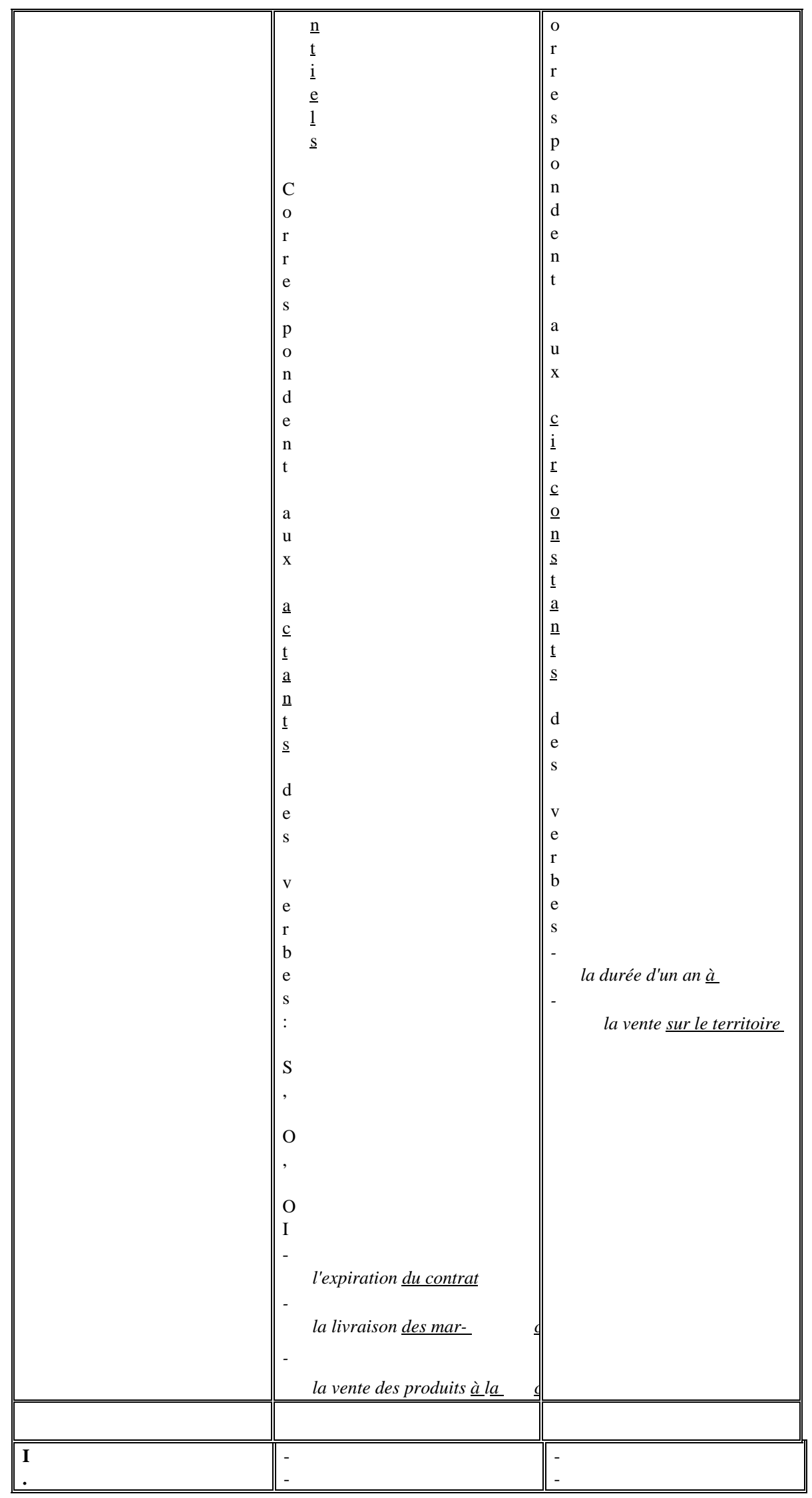




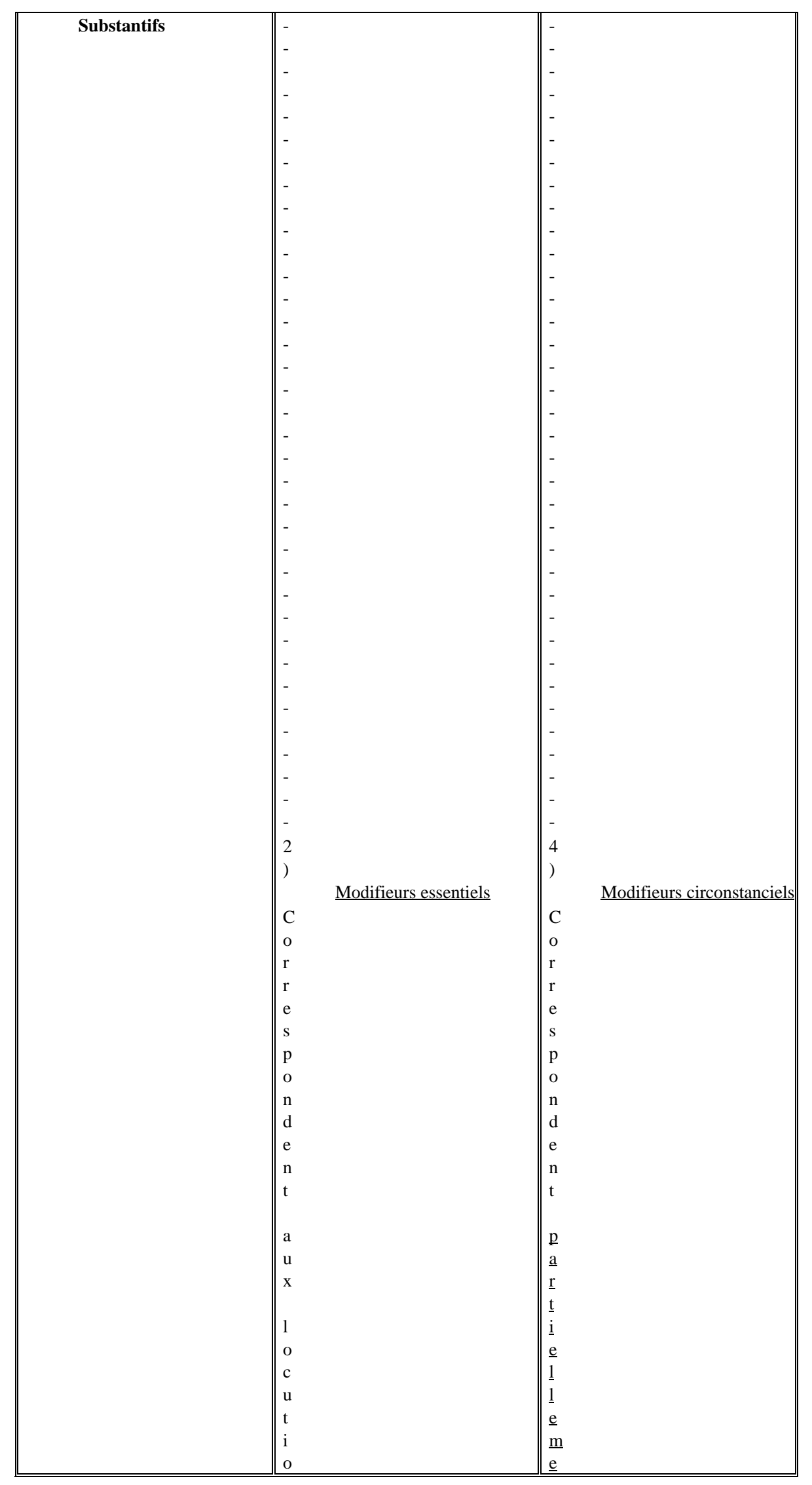




\begin{tabular}{|c|c|c|}
\hline & \begin{tabular}{|ll}
$\mathrm{n}$ \\
$\mathrm{s}$ \\
$\mathrm{a}$ \\
$\mathrm{d}$ \\
$\mathrm{v}$ \\
$\mathrm{e}$ \\
$\mathrm{r}$ \\
$\mathrm{b}$ \\
$\mathrm{i}$ \\
$\mathrm{a}$ \\
$\mathrm{l}$ \\
$\mathrm{e}$ \\
$\mathrm{s}$ \\
\\
$\mathrm{e}$ \\
$\mathrm{s}$ \\
$\mathrm{s}$ \\
$\mathrm{e}$ \\
$\mathrm{n}$ \\
$\mathrm{t}$ \\
$\mathrm{i}$ \\
$\mathrm{e}$ \\
1 \\
1 \\
$\mathrm{e}$ \\
$\mathrm{s}$
\end{tabular} & \begin{tabular}{|ll}
$\underline{\mathrm{n}}$ & \\
$\mathrm{t}$ & \\
$\mathrm{a}$ & \\
$\mathrm{u}$ & \\
$\mathrm{x}$ & \\
$\mathrm{a}$ & \\
$\mathrm{d}$ & \\
$\mathrm{v}$ & \\
$\mathrm{e}$ & \\
$\mathrm{r}$ & \\
$\mathrm{b}$ & \\
$\mathrm{e}$ & \\
$\mathrm{s}$ & \\
- & \\
- & les ventes $\underline{\text { directes sur }}$ \\
- & les frais $\underline{\text { de transport }}$ \\
- & les commandes passées \\
- & toute condamnation,
\end{tabular} \\
\hline Substantifs non & 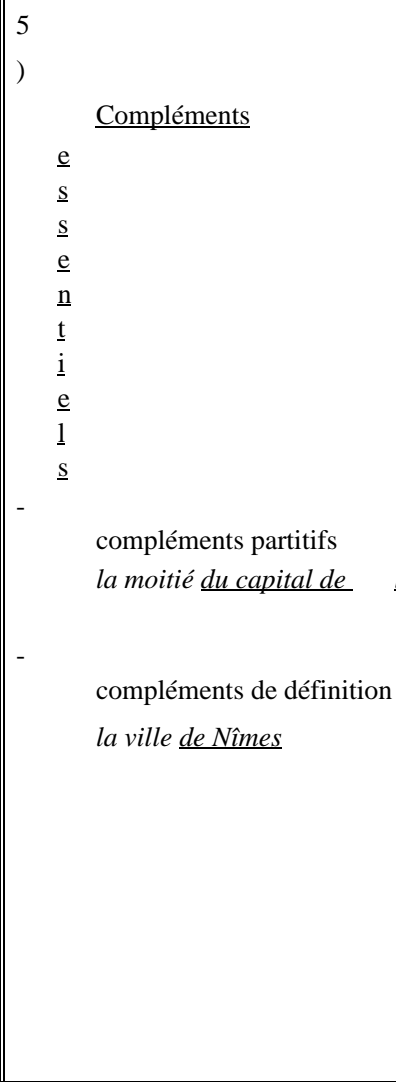 & 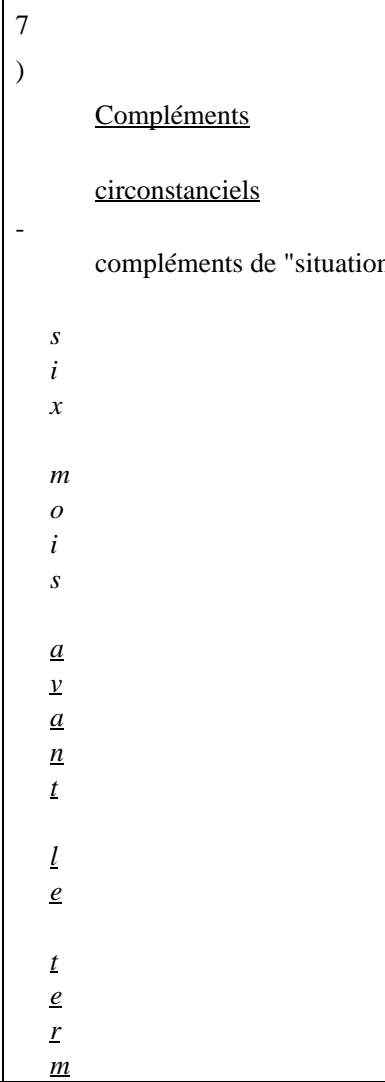 \\
\hline
\end{tabular}




$$
\mathbb{H}
$$




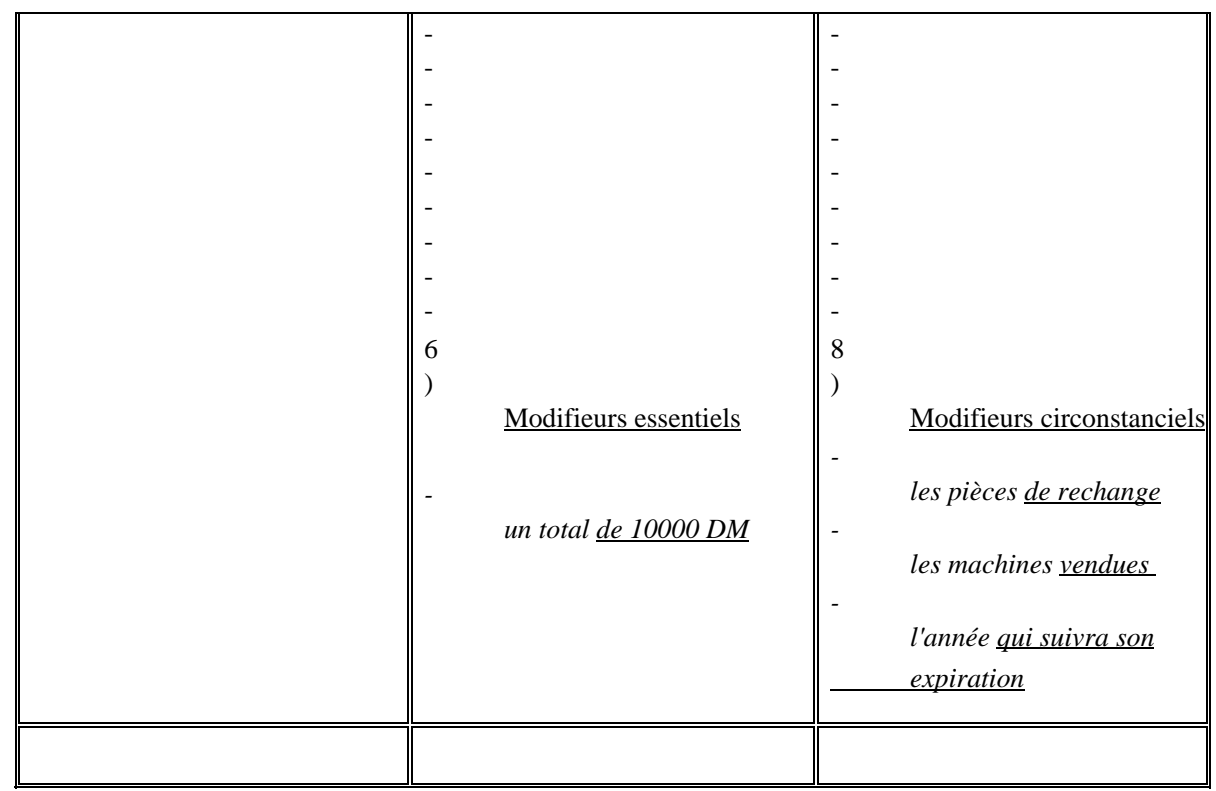

Tableau 2

Bien que compléments et modifieurs essentiels et circonstanciels puissent se combiner entre eux, qu'il s'agisse de substantifs prédicatifs ou non prédicatifs, les substantifs prédicatifs auront virtuellement le plus grand nombre d'expansions. Nous avons vu en effet que leurs compléments essentiels - S, O, OI - qui sont très fréquents, peuvent s'allier presque indéfiniment avec leurs expansions circonstancielles. De plus, les compléments partitifs, de définition et de possession, propres aux substantifs non prédicatifs, ne se rencontrent qu'en nombre limité. Mais qui plus est, tous les syntagmes nominaux pourront contenir, dans leurs expansions, de nouveaux substantifs qui, s'ils sont prédicatifs, admettront à nouveau les mêmes schémas valentiels que les verbes, combinés le cas échéant avec des compléments et des modifieurs circonstanciels. Ainsi, plus on aura de substantifs prédicatifs dans un syntagme et plus on aura, selon toute probabilité, des expansions non seulement horizontales, mais aussi verticales. Nous parlerons, dans ce dernier cas, des différents niveaux de profondeur du syntagme, et de sa structure hiérarchique ou pyramidale. Il va sans dire qu'un autre facteur jouant un rôle décisif quant à ce niveau de profondeur est l'expansion à l'aide de syntagmes participiaux, qui comportent à eux seuls toute une série de compléments et de modifieurs. Or, ce genre d'expansions est particulièrement fréquent dans les textes juridiques, en raison du besoin d'impersonnalité qui les caractérise ${ }^{10}$. C'est ainsi que dans ce type de textes, où le style nominal, avec ses nombreuses expansions participiales, est plus répandu qu'ailleurs, il n'est pas rare de rencontrer des syntagmes comportant, outre les expansions horizontales, cinq, six ou même sept expansions verticales:

1) la distribution (exclusive)

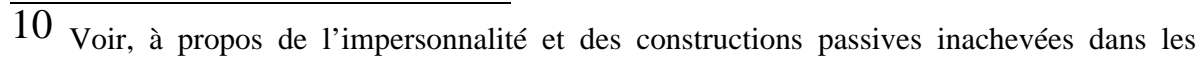
textes juridiques, Sourioux et Lerat $(1975,45)$ et Spilka (1982). 
2) de la vente

3) sur le territoire (français) des produits

4) visés

5) ci-dessous à l'article 2
1) les traites
2) acceptées
3) dès réception
4) des expéditions
5) adressées
6) au concessionnaire

1) un distributeur

2) désigné

3) par le concessionnaire ou par un client

4) situé

5) dans le secteur

6) concédé

7) au concessionnaire

Nous reviendrons, pour clore cette analyse, sur la distinction postulée initialement entre substantifs prédicatifs et substantifs non prédicatifs. Nous avons vu que la caractéristique des premiers était leur faculté d'avoir les mêmes compléments valentiels que les verbes ou locutions verbales dont ils sont dérivés ou avec lesquels ils ont une certaine parenté (exemples types: acceptation, exécution,... qui sont des notions très abstraites). La caractéristique des seconds est leur incapacité à se voir associer ces compléments valentiels (exemples types: magasin, machine,... qui sont des termes très concrets).

Il serait tentant de conclure à un parallélisme systématique entre le caractère prédicatif et le caractère déverbal des substantifs. Or, si un tel parallélisme existe, il est loin d'être systématique. En effet, il s'est avéré que certains substantifs, bien que nous les ayons qualifiés de "dérivés", sont en fait identiques au nom contenu dans une locution verbale à verbe support. D'autres n'ont de verbal que leur parenté avec un verbe. Il est apparu également que certaines formes de nominalisations (les nominalisations subjective et objective) et d'expansions participiales limitaient le nombre de compléments valentiels ou semblaient empêcher leur utilisation. On observe, de plus, que certaines nominalisations subjectives et objectives - donc nettement dérivées de verbes - se rencontrent parfois sans qu'il soit possible de leur associer des compléments valentiels (ex. agent, commerçant, produit). Inversement, les substantifs non prédicatifs concrets pourront, dans certains cas, se voir attribuer un complément subjectif par l'entremise d'un verbe sous-entendu (dénotant l'idée de construction, de fabrication, de création), et ainsi acquérir un plus haut degré d'abstraction (ex. la banque/les machines de la société [c'est-à-dire construite(s) par la société]).

Nous avons souligné, à plusieurs reprises, le caractère plus concret des substantifs privés de certains des compléments valentiels des verbes (ex. le vendeur est perçu comme plus concret que la vente; les achats de la société comme plus concret que l'achat des marchandises). Nous pouvons conclure, 
par conséquent, que moins un substantif a de compléments valentiels, moins il est prédicatif et plus il devient concret.

Il semble bien alors qu'il faille compléter le tableau ci-dessus et le considérer non seulement comme un schéma opérationnel établissant, sur le plan syntaxique, une distinction entre les substantifs prédicatifs et ceux qui ne le sont pas, mais également comme constituant, sur le plan sémantique, une échelle allant du concret vers l'abstrait, avec la possibilité pour les substantifs de se placer sur cette échelle en fonction de leur degré de prédication, c'est-à-dire en fonction $\mathrm{du}$ nombre possible de leurs compléments valentiels.

Plutôt que de parler de parallélisme systématique entre le caractère prédicatif et le caractère déverbal du substantif, il paraît donc plus juste d'établir un parallélisme entre son caractère prédicatif et son niveau d'abstraction. Ce qui confère une dimension nouvelle à notre étude, en ce qu'elle permet de la sorte, outre l'élaboration d'une classification des substantifs et de leurs expansions, de proposer une ébauche de solution à l'épineuse question de la distinction des noms à sens concret et des noms à sens abstrait.

\section{Bibliographie}

Cornu, Gérard (1990): Linguistique juridique. Paris: Montchrestien.

Herslund, Michael (1989): Les relations syntaxiques dans une approche valentielle. Communication au XIXe Congrès International de linguistique et philologie romanes, Saint-Jacques-de-Compostelle (non publiée).

Herslund, Michael / Finn Sørensen (1985): De franske verber. En valensgrammatisk fremstilling: I. Verbernes syntaks. Institut d'Etudes Romanes, Université de Copenhague

(Deuxième édition: 1990, Samfundslitteratur).

Herslund, Michael / Finn Sørensen (1987): De franske verber 2. En valensgrammatisk fremstilling: II. Klassifikation af verberne. Samfundslitteratur, École des Hautes Études Commerciales de Copenhague. 
Herslund, Michael / Finn Sørensen (1992): Verb valency and Automatic Text Processing. In: Steen Jansen / Jørgen Olsen / Henrik Prebensen / Torben Thrane (eds.): Computational approaches to text understanding. Museum Tusculanum Press, Université de Copenhague, 105-113.

Sourioux, Jean-Louis / Pierre Lerat (1975): Le langage du droit. Paris: PUF.

Spilka, Irène V. (1982): Le passif du législateur. In: Jean-Claude Gémar e.a. (eds.): Langage du droit et traduction. Montréal, 101-108.

Stage, Lilian (1986): Franske substantivers valens. In: Niels Davidsen-Nielsen / Finn Sørensen (eds.): CEBAL 8. Copenhague: Nyt Nordisk Forlag, 204-229.

Tesnière, Lucien (1959): Eléments de syntaxe structurale. Paris: Klincksieck (Deuxième édition: 1976).

Ulland, Harald (1991): Les nominalisations agentive et instrumentale en français moderne. Bergen, Diss.

Wagner, Robert Léon / Jacqueline Pinchon (1962): Grammaire du français classique et moderne. Paris: Hachette. 